\title{
Interventionelle Behandlung des akuten und subakuten Vena-cava-superior-Syndroms
}

\author{
Interventional Treatment of the Acute and Subacute Vena Cava \\ Superior Syndrome
}

Autoren

Institute
J.-P. Kühn' ${ }^{1}$, B. Mensel' ${ }^{1}$ R. Ewert ${ }^{2}$, T. Bollmann²

${ }^{1}$ Institut für Diagnostische Radiologie und Neuroradiologie, Ernst-Moritz-Arndt Universität Greifswald

${ }^{2}$ Institut für Innere Medizin, Abteilung Pulmologie, Ernst-Moritz-Arndt Universität Greifswald eingereicht 11.6.2013 akzeptiert nach Revision 4.7.2013

\section{Bibliografie}

Dol http://dx.doi.org/ 10.1055/s-0033-1344490

Online-Publikation: 2.9.2013

Pneumologie 2013; 67: 573-579

(c) Georg Thieme Verlag KG

Stuttgart $\cdot$ New York

ISSN 0934-8387

\section{Korrespondenzadresse} Dr. med. Jens-Peter Kühn Institut für Diagnostische Radiologie und Neuroradiologie Ernst-Moritz-Arndt Universität Greifswald

Sauerbruchstr. 1

17489 Greifswald

kuehn@uni-greifswald.de

\section{Zusammenfassung \\ $\nabla$}

Hintergrund: Infolge eines fehlenden zentralvenösen Abflusses über die obere Hohlvene kann das Vena-cava-superior-Syndrom (VCSS) eine lebensgefährliche Situation darstellen. Ein schnelles und effektives Therapiekonzept ist entscheidend.

Methode: Anhand von zwei Fallberichten wird die endovaskuläre Stenttherapie zur Behandlung des akuten und subakuten VCSS erläutert und diskutiert.

Ergebnisse: Im ersten Fall berichten wir über einen Patienten mit akuter Dyspnoe durch eine obere Einflussstauung bei Dekompensation einer chronischen Thrombose der Vena cava superior. Im zweiten Fall mit gleicher Akutsymptomatik lag eine tumorbedingte Kompression der Vena cava superior vor. Die Akutsituation der VCSS konnte bei beiden Patienten mittels Stentimplantation therapiert werden.

Schlussfolgerung: Die endovaskuläre Therapie des akuten VCSS stellt eine effektive Therapieoption dar.

\section{Einleitung \\ $\nabla$}

Das Vena-cava-superior-Syndrom (VCSS) ist ein Symptomkomplex, der durch eine akute beziehungsweise subakute Komponente einer chronischen Abflussbehinderung der oberen Hohlvene hervorgerufen wird. Pathophysiologisch liegt in fast $90 \%$ der Fälle eine externe Kompression durch einen malignen mediastinalen Tumor vor [1]. In der Mehrzahl der Fälle ist ein Bronchialkarzinom für die Entwicklung ursächlich [2-4]. Als wichtigste nichtmaligne Ursache ist die intraluminale Okklusion durch Thrombosen, zumeist im Rahmen von Venenverweilkathetern oder Schrittmacherkabeln, zu erwähnen, eine tuberkulöse Mediastinitis, fibrosierende Veränderungen, retrosternale Struma sowie posttraumatische

\section{Abstract \\ $\nabla$}

Background: Superior vena cava syndrome is defined as the lack of central venous inflow through the superior vena cava and can present a lifethreatening situation. The acute situation is characterized by dyspnea and requires a fast and effective treatment.

Methods: Using two case reports, endovascular stent therapy for the treatment of acute and subacute superior vena cava syndrome is explained and discussed.

Results: In the first case, we introduce a patient with acute dyspnea due to decompensation of a chronic thrombosis of the superior vena cava. The second case displayed the same acute symptoms consisting of acute dyspnea and upper venous congestion due to a tumor-related compression of the superior vena cava. In both cases, the acute situation of superior vena cava syndrome was successfully treated by stent implantation.

Conclusion: Endovascular treatment of superior vena cava syndrome is an effective option with a high technical success rate.

Strikturen werden in Einzelfällen beschrieben [5 $-7]$.

Patienten mit einem klinisch manifesten VCSS werden durch eine obere Einflussstauung mit einem Ödem der oberen Thoraxapertur, der oberen Extremitäten sowie dem Hals- und Gesichtsbereich auffällig. Klinisch bedeutsam ist eine progrediente Dyspnoesymptomatik.

Die Beeinträchtigung des venösen Rückstromes aus dem Stromgebiet der Vena cava superior kann, aufgrund eines Glottisödems, zu akuten Notfallsituationen führen, in denen ein schnelles interdisziplinäres Therapiekonzept und eine umgehende suffiziente Behandlung der Ursache erforderlich ist. Unerlässlich ist eine symptomatische Therapie mit Sauerstoff, Opiaten, Diuretika, Glukokortikoiden, Lagerung des Patienten in sit- 

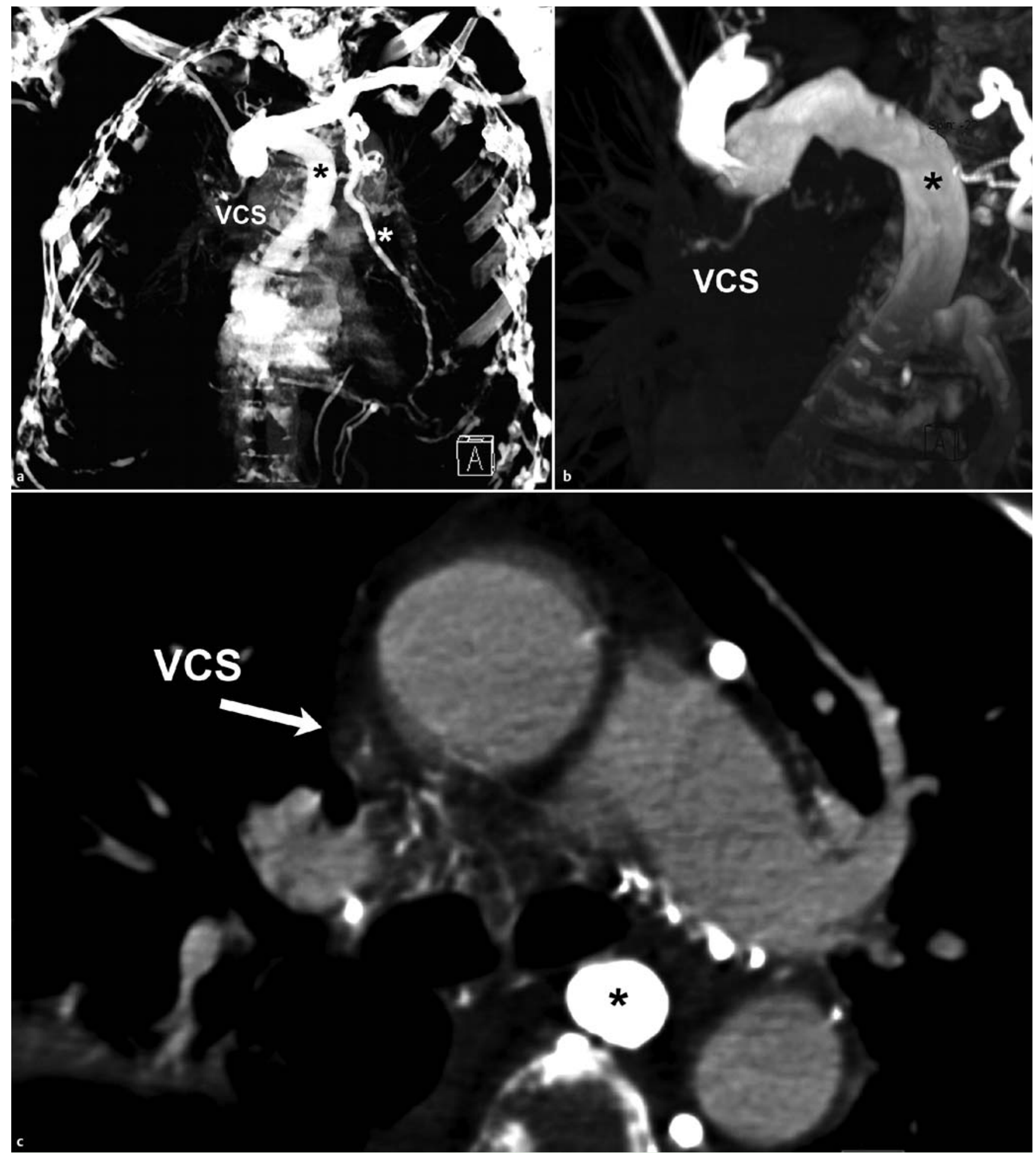

Abb.1 a, b CT-Angiografie mit Kontrastmittelgabe über den linken Arm. Es zeigt sich ein Verschluss der Vena cava superior (VCS) und ein kräftiger Kollateralfluss über die Vena azygos (schwarzer Stern), die linke Vena mammaria (weißer Stern) und perikardiale Venenkonvolute. c Die axiale Schnittführung visualisiert den einen Verschluss der Vena cava superior (VCS).

zender Position etc. [7]. Eine Therapie der Grunderkrankung bei Patienten mit VCSS benötigt häufig Zeit und kann zur Behandlung einer Notfallsituation nur bedingt beitragen. In der Notfallbehandlung des VCSS spielt zum Beispiel die chirurgische Therapie eine eher untergeordnete Rolle.
In dieser Arbeit werden zwei Patienten mit dem seltenen akuten bzw. subakuten Vena-cava-superior-Syndrom differenter Ätiologie vorgestellt und der aktuelle Stand der interventionellen Stent-Therapie zur Primärbehandlung in der Akutsituation diskutiert. 


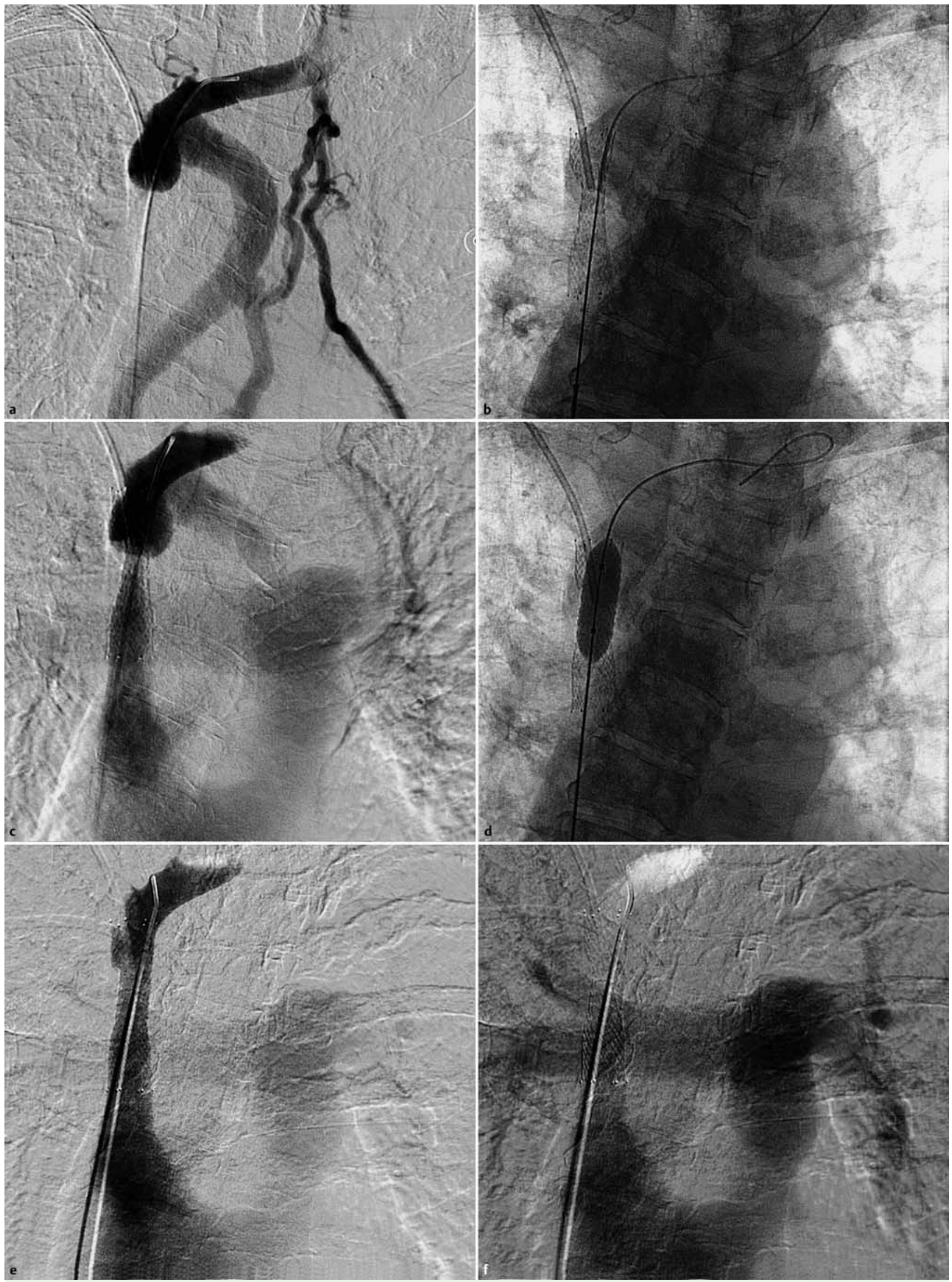

Abb.2 a Sondierung des Verschlusses und präinterventionelle Darstellung mit Nachweis ausgedehnter venöser Kollateralen. b Platzierung eines selbstexpandierbaren Stentsystems, $\mathbf{c}$ Erneute Kontrastmitteldarstellung mit insuffizientem zentralen Abfluss. d Vollständige Aufdehnung des Stentsystems mittels Ballondilatation. e, $\mathbf{f}$ In der Kontrolldarstellung suffizienter Abfluss des Kontrastmittels in den rechten Vorhof, den Truncus pulmonalis und den zentralen pulmonalarteriellen Gefäßen. 


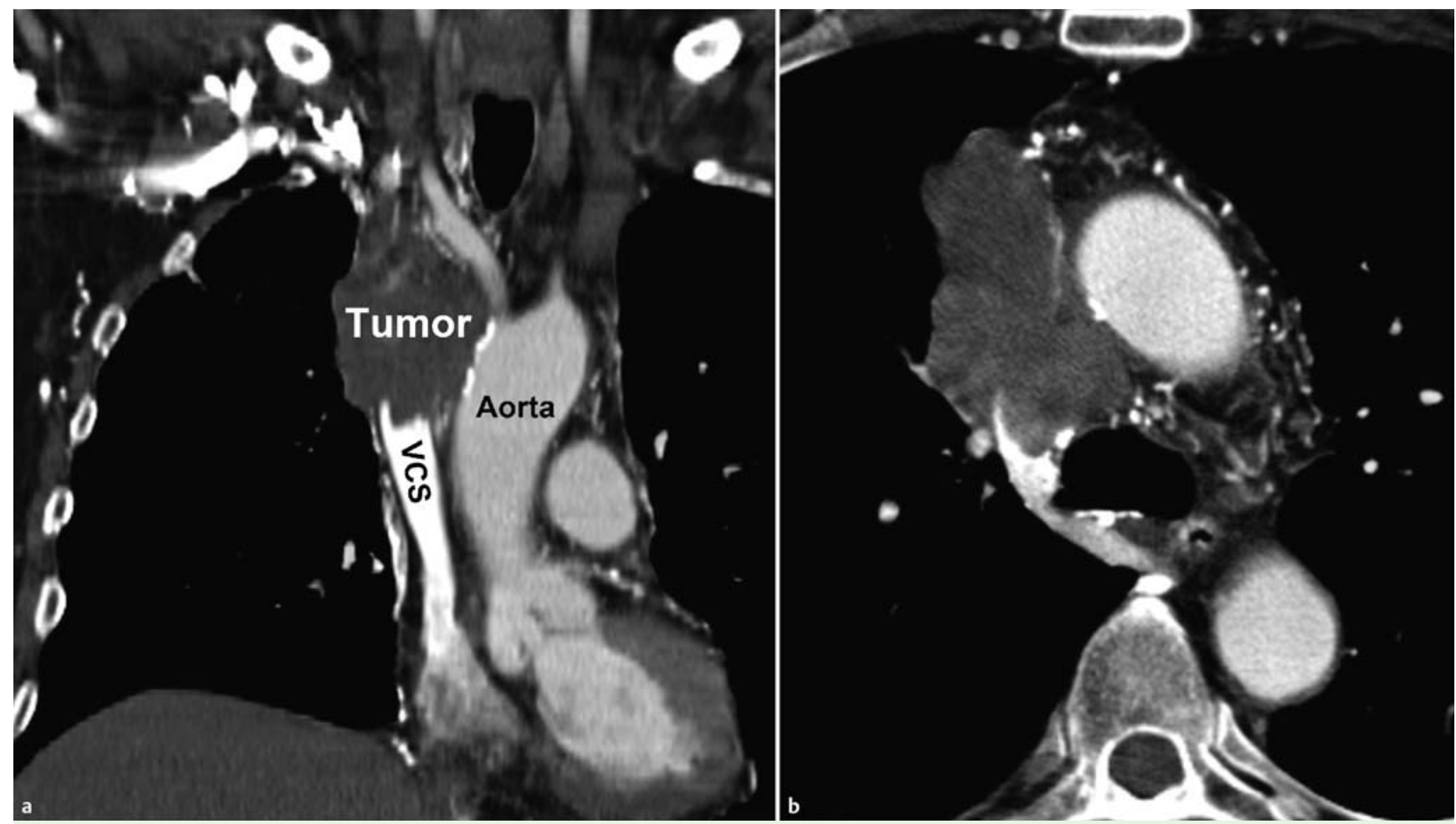

Abb.3 a CT-Angiografie mit Darstellung einer koronaren multiplanaren Reformatierung eines mediastinalen Tumors (T4-Bronchialkarzinoms) mit Infiltration und vollständiger Okklusion der Vena cava superior (VCS). b In der axialen Schnittführung wird ein eher spärlicher Kollateralfluss über perikardiale Venen und die Venae mammariae beobachtet.

Kasuistik 1: Wir berichten über eine 50-jährige weibliche Patientin, mit hepatisch und ossär metastasiertem Mammakarzinom mit palliativem Therapiekonzept, welche sich mit der Symptomatik einer akuten Dyspnoe in der Notfallambulanz vorstellte. Die im guten Allgemein- und adipösen Ernährungszustand befindliche Patientin berichtete über eine Portimplantation vor 4 Jahren und eine, zur Zeit laufende, Chemotherapie. Anamnestisch klagte sie über eine progrediente Schwellung beider Arme, des Halses und Gesichtes. Die Patientin begründete die Schwellung bislang durch die laufende Chemotherapie. Bei laborchemisch positivem D-Dimer von $2,44 \mathrm{mg} / \mathrm{l}$ (Normbereich $0-0,5 \mathrm{mg} / \mathrm{l}$ ) erfolgte zum Ausschluss einer Lungenarterienthrombembolie eine CT-Pulmonalisangiografie. In dieser konnte eine Lungenarterienthrombembolie ausgeschlossen werden. Es zeigte sich ein ausgedehnter venöser Kollateralkreislauf der oberen Thoraxapertur und weitgestellte zervikale Venen bei einem Verschluss der Vena cava superior ( Abb.1). Ein mediastinaler Tumor ließ sich nicht nachweisen. Die angegebene Klinik und der bildmorphologische Verschluss der oberen Hohlvene waren am ehesten durch die Dekompensation einer chronischen Vena-cavasuperior-Thrombose durch das einliegende Portsystem zu erklären. Bei klinisch rapidem Progress der Dyspnoe mit resultierender Zyanose wahrscheinlich in Folge einer zeitgleichen akuten Thrombose der Kollateralen erfolgte die interdisziplinäre Therapieentscheidung zur Durchführung einer Stentimplantation der Vena cava superior ( Abb.2). Die Implantation eines Stentsystems in die Vena cava superior konnte problemlos am Aufnahmetag durchgeführt werden. Die klinische Symptomatik besserte sich schlagartig nach der Stentimplantation. Ein vollständiges Abschwellen der Arme und des Gesichtes war innerhalb der nächste sieben Tage zu beobachten.
Kasuistik 2: Wir berichten über einen 74-jährigen männlichen Patienten mit bekanntem kleinzelligem Bronchialkarzinom rechts (Erstdiagnose 06/2009), welcher sich zur Fortführung seiner Chemotherapie in leicht reduziertem Allgemein- und normosomem Ernährungszustand in der onkologischen Ambulanz vorstellte. Der Patient berichtete über intermittierende Schwellungen der Beine, Arme und seit einer Woche auch eine Schwellung des Gesichtes sowie ausgeprägte Dyspnoe bei leichter Belastung. Klinisch zeigte er eine Zyanose der Lippen, Ohren sowie der Fingerspitzen beidseits. Zusätzlich fiel eine ödematöse Schwellung der Arme und des Gesichtes auf, weiterhin fand sich eine ausgeprägte Venenzeichnung im Sinne venöser Kollateralen auf der oberen Thoraxapertur. Zum Ausschluss einer Thrombose erfolgte zunächst eine venöse Duplexuntersuchung beider Arme, in der sich eine Thrombose der Venae axillares und der Venae subclaviae zeigte. In der Computertomografie wurde der klinische Verdacht eines Progresses des rechts zentralen Bronchialkarzinoms mit progredienter Einengung der Vena cava superior und konsekutiver oberer Einflussstauung und begleitender sekundärer Thrombose bestätigt ( Abb.3). Im interdisziplinären Konsens wurde die Indikation zur interventionellen Stentimplantation vor dem Hintergrund der Fortführung des palliativen Therapieregimes gestellt. Die Stentimplantation konnte nach Aufklärung technisch erfolgreich durchgeführt werden ( $\mathbf{A b b}$.4). Der implantierte Portkatheter wurde bei der Intervention zwischen der Venenwand und dem Stent fixiert, sodass dieser zunächst nicht genutzt werden konnte. Es erfolgte eine postinterventionelle Vollantikoagulation mit niedermolekularem Heparin in therapeutischer Dosierung, die Thrombosen der oberen Extremitäten waren im Verlauf schnell rückläufig. Durch eine Portrevision konnte die Fortsetzung der palliativen Chemotherapie innerhalb der nächsten fünf Tage erfolgen. 


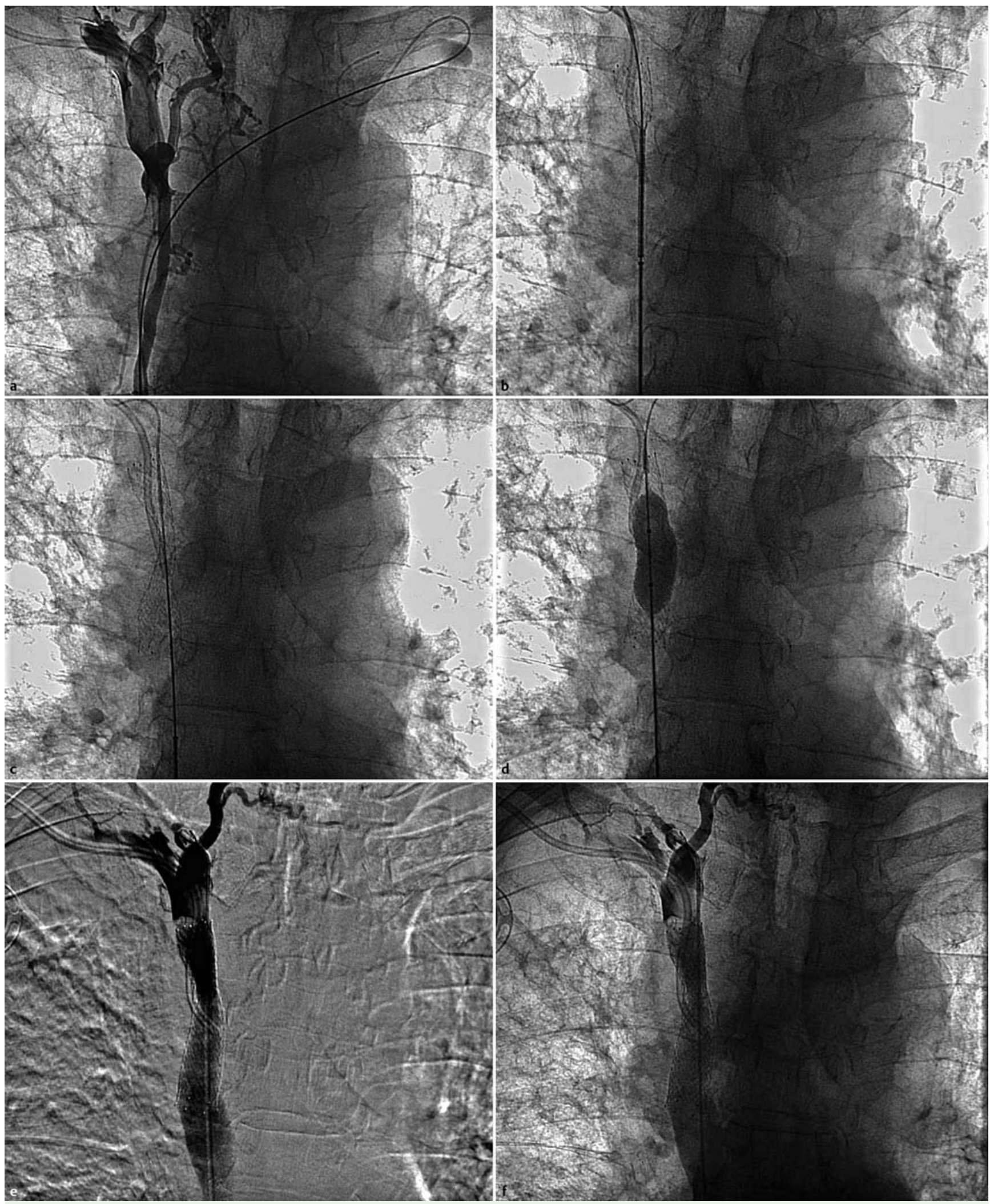

Abb.4 a Sondierung der linken Vena brachiocephalica. Es wird deutlich, dass der Tumor die venöse Bifurkation einbezieht. b Sondierung der linken Vena brachiocephalica und distale Drahtsicherung. Platzierung eines selbstexpandierbaren Stentsystems. c, d Nach vollständiger Entfaltung des Stents wurde dieser mit einer Ballondilatation anmodelliert. e Bei suffizienter Stent-Tumor-Überlappung findet sich thrombotisches Material im Stent und der distalen Vena brachiocephalica. f Ein suffizienter proximaler Zufluss ist gesichert. Im Verlauf wurde der Patient im therapeutischen Bereich vollheparinisiert. Die Beschwerdesymptomatik war schnell rückläufig, und die klinische Erscheinung der oberen Einflussstauung innerhalb von 6 Tagen normalisiert. 


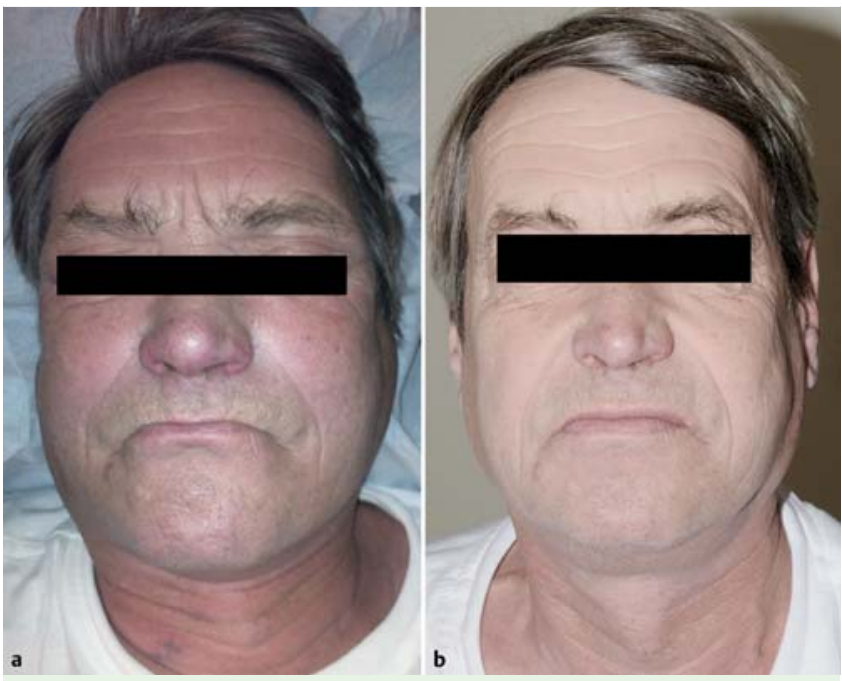

Abb.5 Patient (nicht beschrieben in Kasuistik 1 und 2) mit diffusem Ödem in beiden Armen, Hals und im Gesicht. Bei dem Patienten war ein zentrales Bronchialkarzinom bekannt, welches zu einer Infiltration mit konsekutiver Thrombose der Vena cava superior führte. In palliativer Absicht wurde dem Patienten die Vena cava superior überstentet. a zeigt den klinischen Befund (Gesichtsödem) vor der Intervention. b Nach Stenttherapie zeigte sich eine klinische Besserung mit Normalisierung des Gesichtsödems nach 48 Stunden.

\section{Diskussion}

$\nabla$

Die unterschiedlichen therapeutischen Strategien zur Behandlung des Vena-cava-superior-Syndroms, wie die chirurgische, die medikamentöse und die interventionelle Therapie mit der perkutanen transluminalen Angioplastie, Stenttherapie und thrombolytischem Verfahren, müssen im interdisziplinären Konsens individuell diskutiert werden. Die Wahl des optimalen therapeutischen Konzeptes hängt in entscheidendem Maß von der Grunderkrankung, der Ursache der Obstruktion und der klinischen Gesamtsituation ab. Es muss erwähnt werden, dass im Rahmen der initialen Therapie des Vena-cava-superior-Syndroms zunächst die Behandlung des akuten Symptomkomplexes im Vordergrund steht. Interventionelle Therapieverfahren bieten hierbei eine schonende und häufig sofort wirksame Ergänzung beziehungsweise Alternative zur medikamentösen, radiogenen und chirurgischen Therapie [8-11].

Die primäre Erfolgsrate der interventionellen perkutanen Stenttherapie zur Behandlung des VCSS liegt bei 80 - 95\% der Fälle [12, 13]. In einer der größten publizierten retrospektiven Analysen über einen Zeitraum von 11 Jahren, mit dem Einschluss von 172 Interventionen (24 Okklusionen, 152 höhergradige Stenosen) mit einem Vena-cava-superior-Syndrom, wurden 166 Patienten erfolgreich therapiert. Dies entspricht einer technischen Erfolgsrate von 96,5\% [13]. Nach erfolgreicher Therapie stellte sich eine Linderung der Beschwerdesymptomatik innerhalb der nächsten 6 Stunden ein. Eine vollständige visuelle Besserung der oberen Einflussstauung dauert im Durchschnitt 2-3 Tage $[14,15]$ (৫ Abb.5).

Die Indikation zur perkutanen Stentinsertation zur Behandlung des VCSS bei Patienten mit mediastinalen Malignomen und oberer Einflussstauung stellt ein symptomorientiertes Therapiekonzept zur Linderung der akuten Beschwerdesymptomatik dar. Häufig stehen kausale Therapieoptionen im Rahmen der Therapie eines Rezidives nicht mehr oder nur eingeschränkt zur Verfü- gung. Im Rahmen der Primärtherapie einer malignen Erkrankung kann die interventionelle Versorgung des VCSS in Kombination mit einer im Anschluss durchzuführenden zytostatischen Therapie erwogen werden.

Für Patienten mit einer benignen Obstruktion als Ursache des VCSS sollten zunächst andere Therapieverfahren kritisch diskutiert werden. Absolute Kontraindikationen für Stentimplantationen der zentralen Venen existieren nicht. $\mathrm{Zu}$ den relativen Kontraindikationen zählen eine schlechte Gerinnungsfunktion und eine schlechte Patientencompliance. Ein vollständiger Verschluss oder eine Thrombose der oberen zentralvenösen Strombahn stellt keine Kontraindikation für einen Therapieversuch dar. Liegende Schrittmachersonden sowie funktionstüchtige Herzschrittmachersysteme sollten in situ belassen werden, eine Stentimplantation führt im Regelfall nicht zu einer Funktionsstörung des Aggregats [16,17].

Komplikationen der perkutanen Stenttherapie der Vena cava superior sind sehr selten. Zu den Frühkomplikationen zählen die Stentmigration, die akute In-Stent-Thrombose und die Lungenarterienthrombembolie. Funktionell kann es durch einen sofortigen Anstieg des venösen Rückstromes zu einer akuten Rechtsherzbelastung kommen, in der Literatur ist bislang ein Fall eines plötzlichen Herztodes durch ein akutes Cor pulmonale bekannt. Es existieren keine Langzeitergebnisse der Stenttherapie der Vena cava superior im Rahmen palliativer Therapiekonzepte. Dies ist nicht verwunderlich, zumal die mittlere Überlebensdauer für Patienten mit kleinzelligem bzw. nichtkleinzelligem Bronchialkarzinom im fortgeschrittenen Stadium auch unter laufender Therapie häufig wenige Monate beträgt $[18,19]$. Dennoch ist bei technisch erfolgreicher Durchführung einer Stenttherapie der Vena cava superior für die meisten Malignompatienten nicht erneut mit der Symptomatik des VCSS im Verlauf des restlichen Lebens zu rechnen. Für Patienten mit nicht maligner Ursache des VCSS und erfolgreicher Primärintervention liegen keine ausreichenden Langzeit-Follow-Up-Daten vor. Re-Verschlüsse sind theoretisch denkbar.

\section{Durchführung der Interventionellen Therapie}

Der perkutane Zugangsweg erfolgt über die Leiste, alternative Zugangswege sind beide Jugularvenen. Nach Insertion einer großlumigen Schleuse (z.B. BriteTip ${ }^{\circledR}$ Schleuse, Fa. Cordis, Hamburg, Deutschland) erfolgt eine katheterassistierte Drahtsondierung (z.B. Radifocus ${ }^{\circledR}$ GuideWire M Standard type, Terumo $\mathrm{GmbH}$, Eschborn, Deutschland; 4F Ver135 (Vertebraliskatheter), Fa. Cordis, Hamburg, Deutschland). Dabei muss die Engstelle beziehungsweise der Verschluss in der Vena cava superior vollständig passiert und der Katheter sicher distal platziert werden. Die Thromboseprophylaxe erfolgt durch Bolusinjektion von 5000IE Heparin (Heparin-Natrium-25.000-ratiopharm ${ }^{\circledR}$, ratiopharm $\mathrm{GmbH}$, Ulm, Deutschland). Über den liegenden Katheter wird das Drahtsystem gegen einen steifen Stahldraht (z.B. AmplatzSuperstiff $0.35 / 260 \mathrm{~cm}$, Boston Scientific GmbH, Ratingen, Deutschland) gewechselt. Über den steifen Draht wird das unentfaltete Stentsystem (z. B. S.M.A.R.T ${ }^{\circledR}$ Control $^{\circledR}$, Fa. Cordis, Hamburg, Deutschland) platziert. Eine vorsichtige Ballondilatation (z.B. Suposi MAXI-LD, Fa. Cordis, Hamburg, Deutschland) kann dazu erforderlich sein. Bei Patienten mit frischer thrombotischer Komponente sollte die perkutane Ballondilatation aufgrund des erhöhten Embolierisikos vermieden werden. Eine Überstentung der kontralateralen Vena brachiocephalica ist technisch oft unumgänglich und wird im Verlauf durch Kollateralausbildung ausgeglichen [20]. Bei zusätzlichem bilateralem Verschluss der Vena 
brachiocephalica genügt daher auch die Rekanalisierung einer Seite. Nachfolgend wird das Stentsystem durch einen entsprechenden PTA-Ballon nachdilatiert. Im Falle einer malignen Ursache sollte der Stent den Tumor einige Zentimeter überbrücken, ansonsten so kurz wie möglich gewählt werden. In der Literatur mehrfach erfolgreich angewandte selbstexpandierbare Stentsysteme sind der Wall-Stent [21], Palmaz-Stent [22] und GianturcoZ-Stents [23]. Zur Prävention eines thrombotischen Stentverschlusses sollte postinterventionell eine niedrig dosierte Heparinisierung erfolgen. Eine Langzeitantikoagulation ist nicht zwingend erforderlich und stellt im Rahmen der Tumorerkrankung unter Umständen ein deutlich erhöhtes Blutungsrisiko dar [12].

\section{Zusammenfassung}

Die perkutane Stenttherapie zur Behandlung eines akuten VCSS besitzt eine hohe technische Erfolgsrate, bei niedrigem technischem Schwierigkeitsgrad. Die Indikation zur Stentinsertation der Vena cava superior für Patienten mit mediastinalen Malignomen besteht ausschließlich in der symptomatischen Behandlung des VCSS-Symptomenkomplexes mit dem Ziel einer zügigen Linderung der klinischen Symptomatik und einer Verbesserung der Lebensqualität.

\section{Interessenkonflikt}

$\nabla$

Die Autoren geben an, dass kein Interessenkonflikt besteht.

\section{Literatur}

1 Ostler PJ, Clarke DP, Watkinson AF et al. Superior vena cava obstruction: a modern management strategy. Clin Oncol (R Coll Radiol) 1997; 9: $83-89$

2 Armstrong BA, Perez CA, Simpson JR et al. Role of irradiation in the management of superior vena cava syndrome. Int J Radiat Oncol Biol Phys 1987; 13: $531-539$

3 Davenport D, Ferree C, Blake D et al. Radiation therapy in the treatment of superior vena caval obstruction. Cancer 1978; 42: 2600-2603

4 DyetJF, Moghissi K. Role of venography in assessing patients with superior caval obstruction caused by bronchial carcinoma for bypass operations. Thorax 1980; 35: 628-630

5 Sculier JP, Feld R. Superior vena cava obstruction syndrome: recommendations for management. Cancer Treat. Rev 1985; 12: 209-218

6 Cengiz K, Aykin A, Demirci A et al. Intrathoracic goiter with hyperthyroidism, tracheal compression, superior vena cava syndrome, and Horner's syndrome. Chest 1990; 97: 1005-1006
7 Abner A. Approach to the patient who presents with superior vena cava obstruction. Chest 1993; 103: 394S-397S

8 Capek $P$, Cope $C$. Percutaneous treatment of superior vena cava syndrome. AJR Am J Roentgenol 1989; 152: 183-184

9 Kee ST, Kinoshita L, Razavi MK et al. Superior vena cava syndrome: treatment with catheter-directed thrombolysis and endovascular stent placement. Radiology 1998; 206: 187-193

10 Gross CM, Krämer J, Waigand J et al. Stent implantation in patients with superior vena cava syndrome. AJR Am J Roentgenol 1997; 169: 429432

11 Chin DH, Petersen BD, Timmermans $H$ et al. Stent-graft in the management of superior vena cava syndrome. Cardiovasc Intervent Radiol 1996; 19: $302-304$

12 Mathias $K$, Jäger $H$, Willaschek J et al. [Interventional radiology in central venous obstructions. Dilatation - stent implantation - thrombolysis]. Radiologe 1998; 38: 606-613

13 Miller JH, McBride K, Little $F$ et al. Malignant superior vena cava obstruction: stent placement via the subclavian route. Cardiovasc Intervent Radiol 2000; 23: 155-158

14 Dyet JF, Nicholson AA, Cook AM. The use of the Wallstent endovascular prosthesis in the treatment of malignant obstruction of the superior vena cava. Clinical Radiology 1993; 48: 381-385

15 Stock KW, Jacob AL, Proske $M$ et al. Treatment of malignant obstruction of the superior vena cava with the self-expanding Wallstent. Thorax 1995; 50: 1151 - 1156

16 Teo N, Sabharwal T, Rowland E et al. Treatment of superior vena cava obstruction secondary to pacemaker wires with balloon venoplasty and insertion of metallic stents. Eur. Heart J 2002; 23: 1465 - 1470

17 Melikian N, Brookes CI, Rickards AF. Management of superior vena caval obstruction secondary to a pacing wire with percutaneous intravascular stent insertion. Heart 1999; 82: 182

18 Goldstraw P, Crowley J, Chansky K et al. The IASLC Lung Cancer Staging Project: proposals for the revision of the TNM stage groupings in the forthcoming (seventh) edition of the TNM Classification of malignant tumours. J Thorac Oncol 2007; 2: 706-714

19 Shepherd FA, Crowley J, Van Houtte P et al. The International Association for the Study of Lung Cancer lung cancer staging project: proposals regarding the clinical staging of small cell lung cancer in the forthcoming (seventh) edition of the tumor, node, metastasis classification for lung cancer. J Thorac Oncol 2007; 2: 1067-1077

20 Hennequin $L M$, Fade 0 , Fays JG et al. Superior vena cava stent placement: results with the Wallstent endoprosthesis. Radiology 1995; 196: $353-361$

21 Watkinson AF, Hansell DM. Expandable Wallstent for the treatment of obstruction of the superior vena cava. Thorax 1993; 48: 915-920

22 Dodds GA, Harrison JK, O'Laughlin MP et al. Relief of superior vena cava syndrome due to fibrosing mediastinitis using the Palmaz stent. Chest 1994; 106: 315 - 318

23 Rösch J, Uchida BT, Hall LD et al. Gianturco-Rösch expandable Z-stents in the treatment of superior vena cava syndrome. Cardiovasc Intervent Radiol 1992; 15: 319-327 